\title{
Computação Afetiva aplicada à Educação: uma revisão sistemática das pesquisas publicadas no Brasil
}

\author{
Felipe de Morais ${ }^{1}$, Juarez da Silva ${ }^{1}$, Helena Reis ${ }^{2}$, Seiji Isotani ${ }^{2}$, Patricia Jaques ${ }^{1}$ \\ ${ }^{1}$ Programa de Pós-Graduação em Computação Aplicada (PPGCA) \\ Universidade do Vale do Rio dos Sinos (UNISINOS), São Leopoldo - RS - Brasil \\ ${ }^{2}$ Instituto de Ciências Matemáticas e Computação (ICMC) \\ Universidade de São Paulo (USP), São Carlos - SP - Brasil \\ felipedemoraisfmehotmail.com, juarezcell@gmail.com, \\ \{helenamcd, sisotani\}@icmc.usp.br, pjaques@unisinos.br
}

\begin{abstract}
This paper aims to perform a survey of the scientific work carried out in the last 15 years in the area of Affective Computing in the context of Computers in Education. For this, a systematic mapping was carried out in the main scientific databases of the area in Brazil, being the SBIE, WIE and RBIE, which are events and journal of CEIE. Based on six research objectives, 53 articles were selected. After being analyzed, according to previously defined inclusion and exclusion criteria, a total of 24 articles were selected. From these, the data was mined, aiming to answer the research questions of this work. As a result, the current state of art in Affective Computing was presented. In addition, it was presented the Affective Socio-Dimensions taken into consideration in the selected papers, a map containing the types of studies and the research objectives and, finally, the presentation of the main authors and research groups in the area of Affective Computing in Brazil.
\end{abstract}

Resumo. Este artigo tem como objetivo realizar um levantamento dos trabalhos científicos brasileiros, realizados nos últimos 15 anos, na área da Computação Afetiva no contexto de Informática na Educação. Para isso, foi realizado um mapeamento sistemático nos principais veículos brasileiros de divulgação científica na área, sendo eles o SBIE, WIE e a RBIE, veículos mantidos pela Comissão Especial de Informática na Educação da SBC. Inicialmente, foram coletados 53 artigos, dos quais 24 foram selecionados para análise. Como resultado, foi apresentado o atual estado da arte em Computação Afetiva em relação aos estados afetivos e outros fenômenos socioafetivos levados em considerações nos trabalhos. Também foram desenvolvidos um mapa contendo os tipos de estudos e os objetivos de pesquisa e uma apresentação dos principais autores e grupos de pesquisas na área da Computação Afetiva aplicada à Educação no Brasil.

\section{Introdução}

Os estados afetivos exercem um papel importante na aprendizagem e nos processos cognitivos desenvolvidos pelos estudantes, tais como criatividade, tomada de decisão e memorização [Pekrun 2011]. As emoções podem tanto acelerar como perturbar os processos cognitivos e a aprendizagem [Piaget 1989]. Quando o estudante obtém sucesso na realização 
VI Congresso Brasileiro de Informática na Educação (CBIE 2017)

Anais do XXVIII Simpósio Brasileiro de Informática na Educação (SBIE 2017)

da tarefa, emoções positivas, como engajamento, curiosidade e orgulho, podem promover o aprendizado [Fredrickson 1998]. No entanto, quando ele se encontra bloqueado ou erra, emoções mais negativas, como confusão, podem surgir. Quando o estudante não consegue voltar ao equilíbrio, experimentando emoções positivas, isso pode o levar a um círculo vicioso de emoções negativas (e.g. tédio ou frustração) [Graesser and D'Mello 2011], o que exige intervenção dos tutores humanos.

Esses tutores são eficientes em adaptar suas estratégias aos estados afetivos do estudante [Lehman and et al. 2008], impactando positivamente no processo de ensinoaprendizagem [Graesser and D'Mello 2011, Pekrun 2011]. Dessa forma, é importante que tutores artificiais, como os Sistemas Tutores Inteligentes (STI) e Ambientes Virtuais de Aprendizagem (AVA), também possuam essa habilidade. Para que um ambiente computacional de aprendizagem possa adaptar suas estratégias de ensino aos estados afetivos do aluno (por exemplo, emoção, humor, personalidade, etc), ele deve possuir, no mínimo, as seguintes capacidades: (1) detectar os estados afetivos dos estudantes [Conati 2011, D'Mello and Calvo 2013] e (2) adaptar as suas estratégias cognitivas e afetivas de acordo com os estados afetivos do estudante [Calvo and D'Mello 2011, Arroyo and et al. 2014].

Estudos sobre a detecção e a regulação dos estados afetivos dos estudantes vêm sendo realizados desde os anos 2000 no Brasil. De fato, algumas revisões bibliográficas e tutoriais foram escritos em veículos nacionais de divulgação científica no tema de computação afetiva aplicada à educação [Jaques and Nunes 2012, Jaques et al. 2012, Quarto et al. 2014, Reis and et al. 2015]. No entanto, esses artigos envolvem revisões bibliográficas (não realizam uma revisão sistemática como os mapeamentos sistemáticos ou as meta-análises), além de serem voltadas ao quadro internacional de pesquisa na área. Com o intuito de apresentar uma visão geral sobre as pesquisas em computação afetiva no contexto brasileiro de informática na educação, foi realizado um mapeamento sistemático ${ }^{1}$ das pesquisas existentes nos veículos brasileiros oficiais de divulgação científica na área, mantidos pela Comissão Especial de Informática na Educação (CEIE) da Sociedade Brasileira de Computação (SBC), sendo eles o Simpósio Brasileiro de Informática na Educação (SBIE), o Workshop de Informática na Escola (WIE) e a Revista Brasileira de Informática na Educação (RBIE). O objetivo deste artigo é analisar as pesquisas na área de computação afetiva, realizadas nos últimos 15 anos, para identificar quais as ações de regulação que os ambientes de aprendizagem vêm utilizando, quais os tipos de ambientes de aprendizagem que consideram os estados afetivos dos alunos e quais são esses estados afetivos e como eles são detectados. Além disso, espera-se identificar pesquisadores e grupos de pesquisa na área bem como futuras oportunidades de pesquisa.

\section{O Processo do Mapeamento Sistemático}

O mapeamento conduzido foi baseado no processo descrito por [Petersen et al. 2008], no qual há cinco passos essenciais a serem seguidos: (i) definição de questões de pesquisa, (ii) realização da pesquisa de estudos primários relevantes, (iii) triagem dos documentos, (iv) keywording dos resumos, e (v) a extração de dados e mapeamento.

Considerando que as questões de pesquisa devem exemplificar os objetivos do

\footnotetext{
${ }^{1} \mathrm{O}$ mapeamento sistemático é uma metodologia que envolve a busca por literatura a fim de verificar a natureza, a extensão e a quantidade de estudos publicados (chamados de estudos primários) [Petersen et al. 2008].
} 
VI Congresso Brasileiro de Informática na Educação (CBIE 2017)

Anais do XXVIII Simpósio Brasileiro de Informática na Educação (SBIE 2017)

estudo de mapeamento, as seguintes questões foram elaboradas:

$\mathbf{Q P}_{1}$ : Quais são os estados afetivos considerados?

$\mathbf{Q P}_{2}$ : Como esses estados afetivos são detectados ${ }^{2}$ ?

$\mathbf{Q P}_{3}$ : Quais os tipos de ambientes computacionais empregados?

$\mathbf{Q P}_{4}$ : De que forma os ambientes se adaptam aos estados afetivos dos estudantes?

QP $\mathbf{P}_{5}$ : Os estudos apresentam avaliação empírica? Qual tipo ${ }^{3}$ ?

QP 6 : Quais os pesquisadores e grupos de pesquisa em Informática na Educação têm trabalhado no tema de computação afetiva no Brasil?

O processo de seleção dos artigos contou com três etapas de filtro. Estas etapas foram definidas para garantir que somente estudos da área de computação afetiva fossem contemplados para o mapeamento sistemático proposto neste artigo. As três etapas foram descritas como (i) busca por palavra chave, (ii) leitura dos artigos e (iii) verificação de equivalência entre artigos.

Na primeira etapa foi realizada a busca por artigos que apresentavam em seus títulos as raízes das palavras-chave afe, aff, emo, mot, humo, sent, perso, cog e adapt. Foram usadas somente as raízes das palavras por elas englobarem as variações das palavras nos idiomas inglês e português. Por exemplo, emo representa as variações de emoção, emotion etc. A busca por artigos foi realizada nos seguintes veículos brasileiros de divulgação científica em Informática na Educação: Simpósio Brasileiro de Informática na Educação (SBIE), Workshop de Informática na Escola (WIE) e Revista Brasileira de Informática na Educação (RBIE).

As buscas foram conduzidas durante o mês de junho de 2017, e foram selecionados artigos a partir de 2001. O resultado da etapa de buscas por palavras-chave pode ser observado na Figura 1. A primeira etapa retornou um total de 53 artigos, onde cada um dos artigos encontrados foi recuperado para análise na segunda etapa.

A segunda etapa contou com a leitura dos estudos na íntegra, com a finalidade de identificar os trabalhos que se adequassem ao objetivo deste mapeamento. Nessa etapa, foram excluídos artigos que não apresentam estudos referentes à computação afetiva na educação.

A terceira, e última etapa de seleção, contou com 27 artigos selecionados pela etapa anterior ${ }^{4}$. Nesta fase de seleção, os artigos foram lidos novamente, aplicando os seguintes critérios de exclusão e inclusão, respectivamente:

- Se vários artigos relatam o mesmo estudo, sem avaliações, modelos ou resultados diferentes, apenas o artigo mais recente foi selecionado. Isso engloba artigos de um mesmo grupo de pesquisa de um único trabalho, se os artigos não apresentarem resultados ou contribuiçõos diferentes.

- Se o artigo descreve mais de um estudo, cada estudo foi avaliado individualmente.

\footnotetext{
${ }^{2}$ De que maneira a detecção foi realizada pelo sistema, como por exemplo, comportamento observacional, sensores fisiológicos, entre outros.

${ }^{3} \mathrm{~A}$ avaliação empírica foi realizada por meio de experimentos, estudos de casos, entre outros.

${ }^{4}$ A lista completa de artigos pode ser visualizada em https : / / goo.gl/42ZDyt.
} 
VI Congresso Brasileiro de Informática na Educação (CBIE 2017)

Anais do XXVIII Simpósio Brasileiro de Informática na Educação (SBIE 2017)

Dos 27 artigos resultantes da segunda etapa, 3 artigos foram desclassificados ${ }^{5}$ por relatarem o mesmo estudo, conforme o critério de exclusão da terceira fase. Assim, a terceira etapa retornou como resultado 24 artigos, sendo estes os trabalhos selecionados para a realização deste mapeamento sistemático. A Figura 1 ilustra os artigos selecionados e não selecionados por ano e base. É possível observar que os anos de 2008 e 2015 possuem o maior número de publicações referentes ao tema, seguidos de 2009.

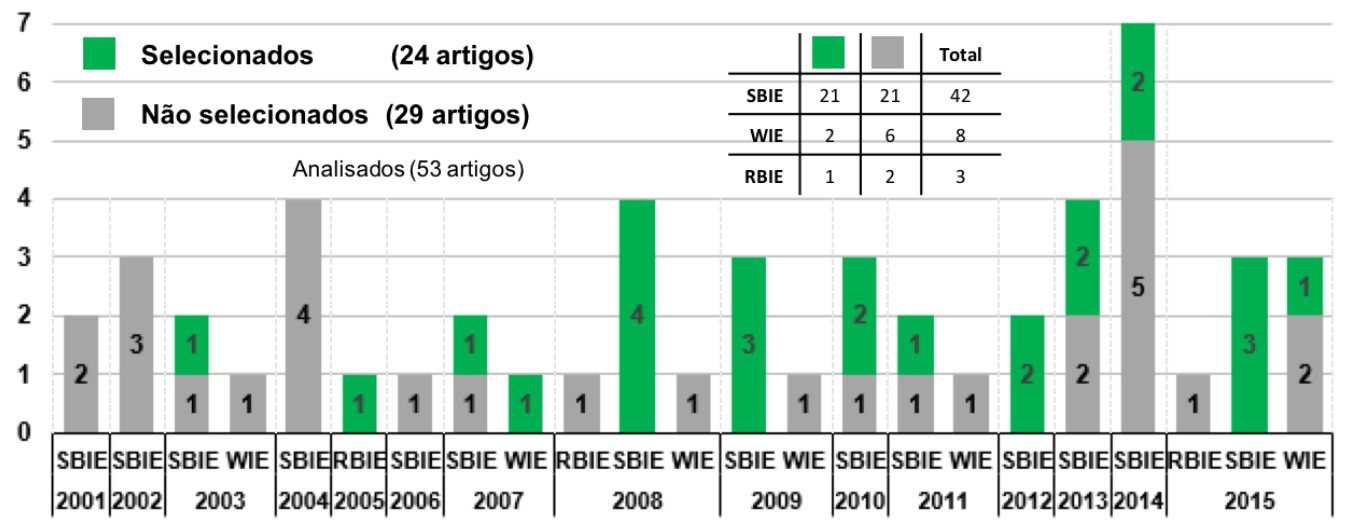

Figura 1. Seleção de estudos por ano e base

Os 24 artigos selecionados passaram por um novo processo de leitura para a extração de informações capazes de classificar os estudos em análise. Esta extração classificada teve como objetivo de busca os seguintes pontos: (i) classificar os estados afetivos e a teoria e/ou modelo utilizado $\left(\mathbf{Q P} \mathbf{P}_{\mathbf{1}}\right)$, (ii) apresentar o método de detecção $\left(\mathbf{Q P} \mathbf{P}_{2}\right)$, (iii) classificar o tipo de ambiente e a fonte do dado $\left(\mathbf{Q P} \mathbf{P}_{\mathbf{3}}\right)$, (iv) apresentar o tipo de regulação do estado afetivo, se houver $\left(\mathbf{Q P}_{\mathbf{4}}\right)$, (v) apresentar o tipo de avaliação empírica empregado, se houver $\left(\mathbf{Q P}_{\mathbf{5}}\right)$, e (vi) apresentar os pesquisadores e grupos de pesquisa $\left(\mathbf{Q} \mathbf{P}_{\mathbf{6}}\right)$. Os resultados obtidos foram utilizados para embasar as respostas das questões de pesquisa deste mapeamento, descritas na Seção 3.

\section{Resultados e Discussões}

Nesta seção são descritos os resultados obtidos a partir da leitura e classificação dos 24 trabalhos selecionados. Para isso, esta seção está dividida em subseções, as quais cada uma descreve os resultados de acordo com uma questão de pesquisa.

\section{1. $\mathrm{QP}_{1}$ : Estados afetivos considerados}

Nessa etapa o objetivo era identificar quais são os estados afetivos detectados ou considerados pelas pesquisas estudadas. Usamos estado afetivo (ou também fenômenos afetivos, devido a sua natureza dinâmica [Scherer 2005]) no lugar de emoção, pois esse é um termo mais abrangente para designar um estado mental afetivo, que pode ser uma emoção, estado de ânimo (humor), postura interpessoal, preferências afetivas, disposição afetiva (traços de personalidade), etc [Scherer 2001, Scherer 2005]. No entanto, durante o mapeamento foram verificados outros fenômenos socioafetivos, tais como motivação e habilidades socioafetivas, que não se encontram dentro da classificação do Scherer de estados afetivos, mas que vem sendo estudados na área de Computação Afetiva.

\footnotetext{
${ }^{5}$ Tal decisão foi tomada mediante o consentimento de todos os autores deste trabalho
} 


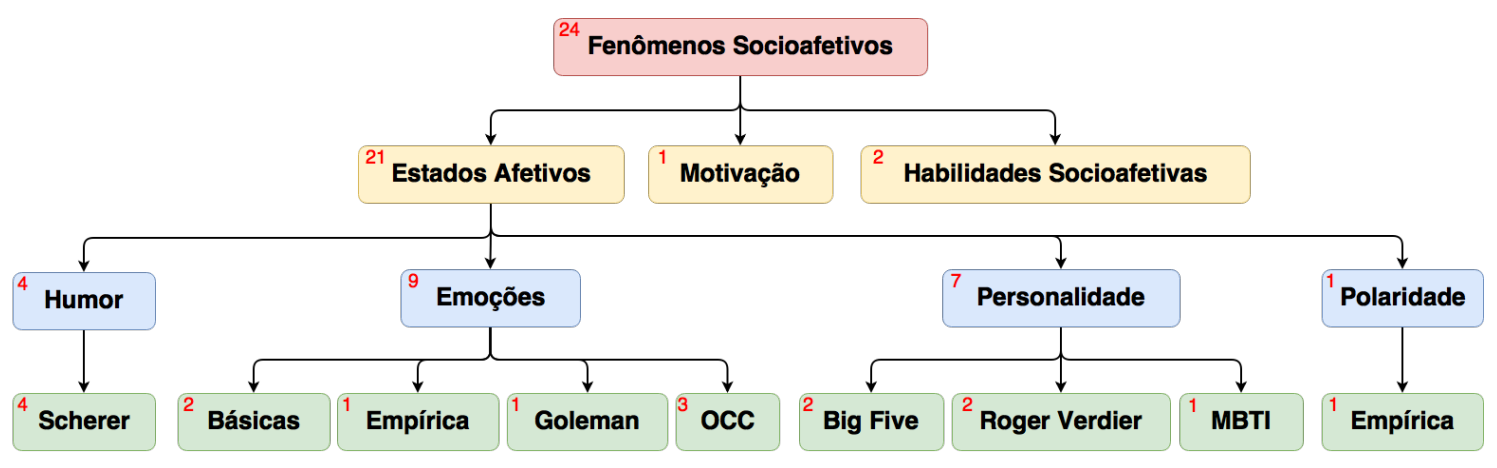

Figura 2. Árvore de fenômenos socioafetivos e as teorias seguidas

A Figura 2 ilustra todos os fenômenos socioafetivos e suas subclasses, identificados na triagem dos resultados, em forma de uma árvore. Além disso, a Figura 2 também exibe o número de trabalhos (em vermelho, no canto superior esquerdo de cada categoria) que considera cada um dos fenômenos. Outra informação contida na árvore é a teoria ou modelo da Psicologia de Emoções que os autores basearam seus trabalhos (retângulos verdes). Na categoria humor, todos os trabalhos identificados eram do mesmo grupo e se basearam no modelo de estado de ânimo de Scherer. Na categoria emoções, dois trabalhos seguiram a teoria das emoções básicas de Ekman, um trabalho usou uma abordagem empírica $^{6}$, um trabalho seguiu a teoria de Goleman, três trabalhos seguiram o modelo OCC e dois trabalhos não identificaram quais teorias usaram. Na categoria personalidade, dois trabalhos seguiram o modelo Big Five, dois trabalhos seguiram a teoria de Roger Verdier, um trabalho seguiu Myers-Briggs Type Indicator (MBTI) e dois trabalhos não identificaram quais teorias seguiram. Na categoria polaridade, um trabalho usou uma abordagem empírica (mineração de dados). Na categoria motivação houve um trabalho e na categoria habilidades socioafetivas houveram dois trabalhos; eles não identificaram as teorias seguidas.

Com base nos resultados ilustrados na Figura 2, é possível identificar que a maior parte dos trabalhos tem levado em consideração os estados afetivos. Mais especificamente, emoções, personalidade e humor são os estados afetivos mais detectados.

\section{2. $\mathrm{QP}_{2}$ : Fonte de dados e ferramentas computacionais usadas na detecção}

Na questão de pesquisa $\mathbf{Q} \mathbf{P}_{2}$, o objetivo era identificar qual a principal fonte de dados empregada para a detecção dos estados afetivos dos alunos, bem como as ferramentas computacionais. As fontes de detecção identificadas nos trabalhos selecionados foram classificadas nas categorias: Comportamento observável, Texto, Escala de personalidade, Base de imagens e expressões faciais e, por fim, Auto-relato.

A categoria Comportamento observável (12 trabalhos) engloba os trabalhos que capturam os estados afetivos através da interação do aluno com a interface do ambiente de aprendizagem, por exemplo, acertar uma tarefa, tempo de resolução de um exercício, número de erros etc. A categoria Texto (7 trabalhos) envolve os sistemas que inferem os estados afetivos através uma fonte textual, podendo ser textos publicados em AVAs,

\footnotetext{
${ }^{6}$ Denominamos abordagem empírica quando o trabalho não está fundamentado em uma teoria psicológica ou filosófica de emoções, mas busca algum tipo de validação empírica. Por exemplo, imagens rotuladas com emoções, classificação por humanos das emoções expressas pelo estudante na face ou no texto, etc.
} 
VI Congresso Brasileiro de Informática na Educação (CBIE 2017)

Anais do XXVIII Simpósio Brasileiro de Informática na Educação (SBIE 2017)

troca de mensagens entre alunos e professores etc. Escala de personalidade (5 trabalhos) se aplica aos trabalhos que utilizam algum tipo de escala (testes de personalidade), tais como Roger Verdier, MBTI etc, não envolvendo algum tipo de detecção automática. A categoria Base de imagens e expressões faciais (2 trabalhos) engloba trabalhos que detectam as emoções através de imagens ou vídeos (webcams). Na categoria Auto-relato ( 2 trabalhos) estão inclusos os trabalhos que perguntam explicitamente ao usuário o seu estado afetivo, geralmente através de uma janela pop-up que é exibida a cada $x$ minutos, ou um botão que o aluno pode pressionar quando sente uma emoção diferente. Dois dos trabalhos não apresentam nenhum tipo de detecção, por isso não foram classificados nas categorias definidas. A soma dos trabalhos não resulta em 24 pois alguns dos trabalhos foram classificados em mais de uma categoria.

É possível, através da análise dos resultados, identificar que comportamento observável, texto e escalas de personalidade são as fontes de dados mais utilizadas para a detecção dos estados afetivos dos alunos nos trabalhos de pesquisa brasileiros.

\section{3. $\mathrm{QP}_{3}$ : Tipos de ambientes}

A questão de pesquisa $\mathbf{Q} \mathbf{P}_{3}$ tem como objetivo mapear os tipos de ambientes utilizados nos estudos que consideram os estados afetivos dos alunos. Os resultados obtidos foram classificados e agrupados em nove tipos de ambientes.

Com base nos resultados obtidos, o ambiente mais utilizado pelos pesquisadores é o Sistema Tutor Inteligente, com 20,8\% (5 casos) de representatividade dos trabalhos analisados. Em segundo lugar, pode ser observado o empate entre o Ambiente Colaborativo de Aprendizagem e o Ambiente Virtual de Aprendizagem com 16,7\% (4 casos) de representatividade cada. A terceira posição, com 12,5\% (3 casos) de representatividade, é ocupada por Objeto de Aprendizagem, seguido pelo Ambiente de Ensino de Programação, com 8,3\% (2 casos), como o quarto mais utilizado. Por fim, os ambientes que levam em consideração os estados afetivos do aluno com 4,2\% (1 caso) de representatividade cada são: Rede Social, Jogo e Base de Imagens. A categoria de Não Especificados, com 12,5\% (3 casos), representa os estudos que não envolveram um ambiente de aprendizagem (por exemplo, detecção de emoções por face usando apenas uma base de dados expressões faciais).

\section{4. $\mathrm{QP}_{4}$ : Formas de adaptação}

O objetivo com a questão de pesquisa $\mathbf{Q P} \mathbf{P}_{4}$ é, dado que os ambientes já identificaram os estados afetivos dos alunos, verificar o que é feito com essa informação, ou seja, como o sistema se adapta baseado no estado afetivo do aluno? Segundo os resultados obtidos, os sistemas utilizam algumas táticas para adaptação, sendo elas, agrupamento de alunos baseado em seu estado afetivo, expressão de emoções através de um agente animado, regulação emocional e mensagens motivacionais. Porém, observa-se que grande parte dos trabalhos são ainda incipientes e os autores não conseguiram mostrar empiricamente que o trabalho atingiu seu objetivo. Além disso, alguns trabalhos apenas descrevem uma proposta de adaptação aos estados afetivos do aluno.

Com o objetivo de melhor compreender o estado da arte de detecção e de adaptação dos ambientes, baseado nos estados afetivos dos alunos, foi elaborado um mapa. Esse mapa, ilustrado pela Figura 3 (formato de bubble chart), tem o objetivo de explicitar a distribuição por tipo de trabalho, expressos no eixo x (baseado nos tipos de estudos definidos 
VI Congresso Brasileiro de Informática na Educação (CBIE 2017)

Anais do XXVIII Simpósio Brasileiro de Informática na Educação (SBIE 2017)

por Petersen [Petersen et al. 2008]) por Avaliação Empírica e Proposta de Trabalho e objetivos de pesquisa, expressos no eixo y por Detecção Automática ou Manual, Expressão e Regulação Detectada ou Presumida. O objetivo Detecção visa identificar de que forma os estados afetivos foram capturados pelos sistemas, de forma manual ou automática. $\mathrm{O}$ objetivo Expressão visa identificar os trabalhos que, de alguma forma, expressam algum tipo de emoção aos alunos, geralmente através de agentes animados. E, por fim, o objetivo Regulação visa identificar os trabalhos que se adaptam ao estado afetivo dos alunos e se essa adaptação é baseada nos estados afetivos detectados ou presumidos pelo sistema (o sistema não detecta o estado afetivo do estudante, mas considera que em uma dada situação, ele sempre sente uma emoção).

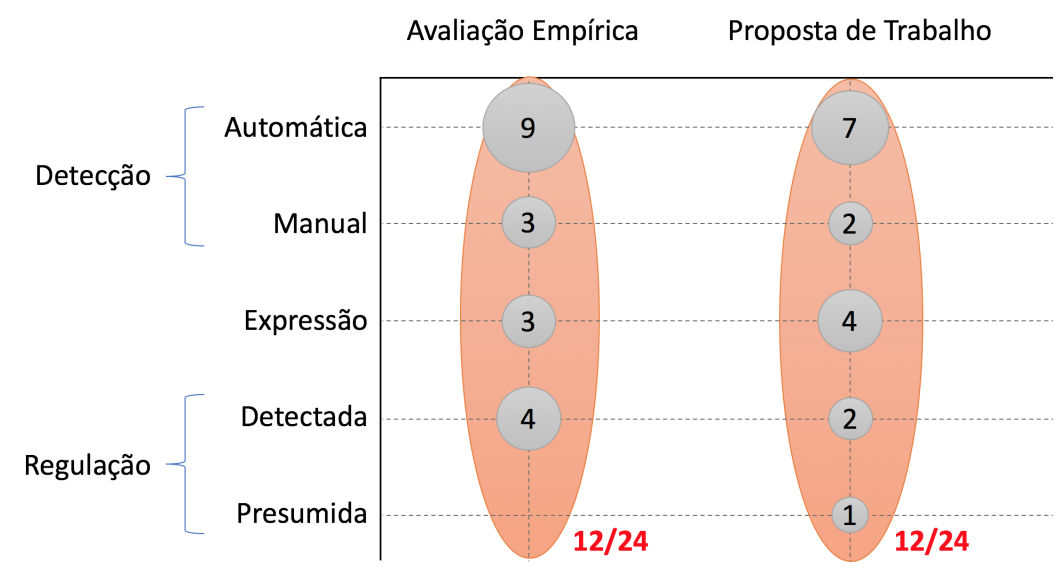

Figura 3. Mapa da distribuição dos trabalhos

No mapa da Figura 3, cada artigo analisado foi classificado em somente um tipo de trabalho (eixo das abscissas). Porém, um trabalho pode possuir mais de um objetivo (eixo das ordenadas), assim o somatório das interseções é maior do que o número de trabalhos. Além disso, os valores, assim como o tamanho dos círculos, na interseções dos eixos das abscissas e ordenadas, representam o número de trabalhos que pertencem a um determinado tipo e possuem determinado objetivo. Com base nos resultados apresentados neste mapa, é possível identificar que apenas sete trabalhos se adaptam aos estados afetivos dos alunos, sendo que destes um trabalho realiza essa adaptação de forma presumida e que três destes trabalhos são apenas propostas, não apresentando algum tipo de avaliação empírica.

\section{5. $\mathrm{QP}_{5}$ Presença de avaliação empírica}

A questão de pesquisa $\mathbf{Q P}_{5}$ visa identificar se os trabalhos apresentaram algum tipo de avaliação empírica e, se sim, quais seriam esses tipos de avaliações.

Novamente, com base nos resultados do mapa da Figura 3, levando em consideração que os estudos se sobrepõem nos resultados do eixo y, as bolhas em destaque representam o número (descritos em vermelho) de estudos únicos em cada tipo de trabalho (eixo das abscissas). Assim, é possível identificar que, dos 24 trabalhos, 12 apresentam algum tipo de avaliação empírica, seja ela qualitativa (por exemplo, observação, entrevistas) ou quantitativa (como experimento, estudos correlacionais, etc). Além disso, 12 trabalhos apresentam apenas uma proposta de trabalho. Dos trabalhos que apresentam avaliação empírica, estas foram identificadas como estudos de casos e experimentos, usando para 
VI Congresso Brasileiro de Informática na Educação (CBIE 2017)

Anais do XXVIII Simpósio Brasileiro de Informática na Educação (SBIE 2017)

análise dos dados estatística descritiva (média, desvio padrão, matriz de confusão, gráficos, etc) e/ou inferência estatística (teste t, ANCOVA, etc).

\section{6. $\mathrm{QP}_{6}$ : Grupos de Pesquisa e Pesquisadores}

A questão de pesquisa $\mathbf{Q} \mathbf{P}_{\mathbf{6}}$ tem como propósito mapear os grupos de pesquisa e os pesquisadores que têm trabalhado com o tema de Computação Afetiva nos últimos 15 anos no Brasil. A Figura 4 (A), em forma de tagcloud, apresenta os pesquisadores autores dos artigos analisados. Na nuvem, são apresentados os autores com pelo menos um artigo selecionado no período analisado. Pode ainda ser observado a representação dos autores neste tema, uma vez que, o tamanho da fonte apresentado na nuvem é diretamente proporcional ao número de publicações.
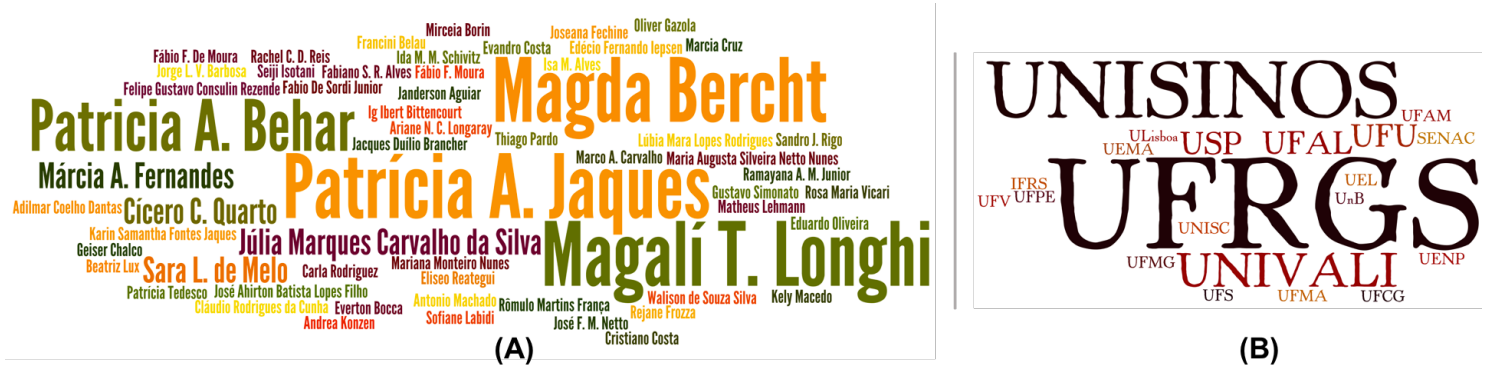

(B)

Figura 4. Tagclouds de autores (A) e grupos de pesquisa (B)

Na Figura 4 (B) podem ser observados os grupos de pesquisa (representados por suas universidades), por entidades de ensino, em forma de tagcloud. Nesta representação entraram os grupos de pesquisa com pelo menos uma publicação na seleção realizada por este trabalho. Como o tamanho da fonte é diretamente proporcional ao número de publicações, pode-se observar a representatividade dos grupos. Ao responder a questão de pesquisa $\mathbf{Q P} \mathbf{P}_{\mathbf{6}}$, chamou atenção o fato de que o estado do Rio Grande do Sul representa um terço das publicações por grupo de pesquisa na seleção de artigos com o tema de Computação Afetiva do Brasil.

\section{Ameaças à Validade}

As questões de pesquisa e os critérios de inclusão e exclusão foram criados antes do início do mapeamento, com a finalidade de garantir um processo de seleção imparcial. A seleção dos artigos foi realizada de forma independente entre os autores, respeitando os critérios de inclusão e exclusão definidos previamente. Ao final, eventuais dúvidas e desacordos foram sanados. Conforme descrito na Seção 2, somente um conjunto limitado de base de dados foi utilizado, portanto é possível que estudos relevantes não tenham sido incluídos. A coerência do sistema de classificação elaborado pode significar uma ameaça à validade, uma vez que o conhecimento necessário para elaborá-lo muitas vezes é obtido somente ao final da seleção [Pretorius and Budgen 2008].

\section{Conclusão}

Este trabalho teve como objetivo realizar um mapeamento sistemático sobre as pesquisas brasileiras na área de Computação Afetiva aplicada à Educação, nos principais veículos de divulgação científica, mantidos pelo CEIE/SBC. Dos 53 artigos levantados das bases 
VI Congresso Brasileiro de Informática na Educação (CBIE 2017)

Anais do XXVIII Simpósio Brasileiro de Informática na Educação (SBIE 2017)

eletrônicas do SBIE, WIE e do RBIE, 24 foram selecionados com base nos critérios de inclusão e exclusão. A partir destes artigos selecionados, foi possível responder às seis questões de pesquisas que guiaram esse trabalho, que dizem respeito a como os trabalhos detectam e respondem às emoções dos estudantes e quais recursos computacionais eles utilizam para tal, além de buscar identificar os principais pesquisadores e grupos de pesquisa na área.

Foi possível identificar que muitos trabalhos brasileiros ainda focam em detectar as emoções básicas [Ekman 1994], respondendo a $\mathbf{Q P}_{\mathbf{1}}$, e a maioria dos trabalhos capturam os estados afetivos através do comportamento observável do aluno ao interagir com o sistema $\left(\mathbf{Q P}_{\mathbf{2}}\right)$. Uma possível explicação é que essa é a forma menos intrusiva de detecção. Além disso, alguns recursos de hardware para detecção, geralmente vendidos no exterior, são bastante caros para o contexto brasileiro. Os Sistemas Tutores Inteligentes representam a maior parte dos ambientes que levam os estados afetivos em consideração $\left(\mathbf{Q P} \mathbf{P}_{\mathbf{3}}\right)$. Muitos dos artigos envolvendo detecção apresentam apenas uma proposta de trabalho, sem uma avaliação empírica.

Poucos trabalhos apresentam algum tipo de adaptação às emoções do aluno. Destes, um pequeno número apresenta algum tipo de avaliação empírica $\left(\mathbf{Q} \mathbf{P}_{\mathbf{5}}\right)$. As principais formas de adaptação são agrupamento de alunos, expressão de emoções por um agente animado, regulação emocional e mensagens motivacionais $\left(\mathbf{Q P}_{\mathbf{4}}\right)$. Por fim, verificou-se, com o uso de tagclouds, quem são os principais pesquisadores e grupos de pesquisas da área de Computação Afetiva do Brasil $\left(\mathbf{Q P}_{\mathbf{6}}\right)$, situando esses principalmente no sul do Brasil.

Baseado nesse estudo, os autores acreditam que há três principais direcionamentos às pesquisas brasileiras de informática na educação para uma contribuição de pesquisa mais relevante na área de computação afetiva. Primeiramente, observa-se que muitas pesquisas brasileiras sobre detecção de estados afetivos ainda se concentram na detecção de emoções básicas. No entanto, já há muitos resultados científicos nesse assunto, assim é difícil propor algo original no tema. Além disso, já foi mostrado que as emoções básicas acontecem raramente no contexto de ambientes de aprendizagem [D'Mello and Calvo 2013]. Dessa forma, indica-se que as pesquisas devem ser direcionadas a outros fenômenos socioafetivos, mais presentes no contexto de aprendizagem, tais como confusão, frustração, flow, etc.

Um outro caminho possível de pesquisa é a regulação das emoções dos estudantes de forma a maximizar a sua aprendizagem. Como há poucas pesquisas na área da educação e psicologia sobre qual a melhor forma de regular as emoções do estudantes para melhorar a sua aprendizagem [Pekrun 2011], há aqui um campo promissor de pesquisa, em que os resultados da computação afetiva podem trazer evidências também para a educação. Por último, mas não menos importante, dos 24 artigos analisados, apenas 12 apresentam algum tipo de avaliação empírica, seja ela tanto qualitativa ou quantitativa. A comunidade brasileira em computação afetiva precisa avançar em relação às propostas de trabalho no sentido de buscar resultados baseados em evidências. Uma pesquisa passa a ser uma contribuição científica apenas quando ela traz evidências que confirmam, ou negam, uma hipótese ou questão de pesquisa (no caso de pesquisas explicativas) ou quando ela traz um estudo qualitativo aprofundado sobre uma questão de pesquisa exploratória. É apenas através da avaliação que esses resultados podem ser obtidos. 
VI Congresso Brasileiro de Informática na Educação (CBIE 2017)

Anais do XXVIII Simpósio Brasileiro de Informática na Educação (SBIE 2017)

\section{Referências}

Arroyo, I. and et al. (2014). A multimedia adaptive tutoring system for mathematics that addresses cognition, metacognition and affect. IJAIED, 24(4):387-426.

Calvo, R. A. and D'Mello, S. K., editors (2011). New Persp. on Affect and LT. Springer, NY, NY.

Conati, C. (2011). Combining Cognitive Appraisal and Sensors for Affect Detection in a Framework for Modeling User Affect. In New Persp. on Affect and LT, pages 71-84. Springer.

D'Mello, S. and Calvo, R. (2013). Beyond the basic emotions. In CHI EA '13, NY. ACM.

Ekman, P. (1994). All Emotions are Basic. In Davidson, R., editor, The nature of emotions: Fundamental questions, pages 15-19. Oxford University Press, Oxford.

Fredrickson, B. (1998). What good are positive emotions. Review of Gen. Psych., 2:300319.

Graesser, A. and D'Mello, S. K. (2011). Theoretical Perspectives on Affect and Deep Learning. In New Persp. on Affect and LT, pages 11-21. Springer, NY, NY.

Jaques, P. A. and Nunes, M. A. S. N. (2012). Ambientes Intel. de Aprendiz. que inferem, expressam e possuem emoções e personalidade. In JAIE, pages 31-82. SBC.

Jaques, P. A., Nunes, M. A. S. N., Isotani, S., and Bittencourt, I. I. (2012). Computação Afetiva aplicada à Educação. In CSBC 2012 - DesafIE.

Lehman, B. and et al. (2008). What Are You Feeling? Investigating Student Affective States During Expert Human Tutoring Sessions. In ITS, pages 50-59, Berlin, Heidelberg. Springer.

Pekrun, R. (2011). Emotions as Drivers of Learning and Cognitive Development. In New Persp. on Affect and LT, volume 3, pages 23-39. Springer, NY.

Petersen, K., Feldt, R., Mujtaba, S., and Mattsson, M. (2008). Systematic mapping studies in software engineering. In EASE, volume 8, pages 68-77.

Piaget, J. (1989). Les relations entre l'intelligence et l'affectivité dans le developpement de l'enfant. In Textes de base en psychologie, pages 75-95. Paris: Delachaux et Niestlé.

Pretorius, R. and Budgen, D. (2008). A mapping study on empirical evidence related to the models and forms used in the uml. In ACM-IEEE ESEM, pages 342-344.

Quarto, C. C., Oliveira, A. N., and Bercht, M. (2014). Agentes Pedagógicos com Dimensões Afetivas: uma tecnologia no apoio ao ensino e a aprendizagem. In SBIE, pages 40-44.

Reis, R. and et al. (2015). Estado da Arte sobre Afetividade na Formação de Grupos em Ambientes Colaborativos de Aprendizagem. RBIE, 23(03):113.

Scherer, K. R. (2001). Appraisal considered as a process of multilevel sequential checking. Appraisal processes in emotion: Theory, methods, research, 92(120):57.

Scherer, K. R. (2005). What are emotions? And how can they be measured? Social Science Information, 44(4):695-729. 